\title{
Kinetic analysis and mathematical modeling of growth and lactic acid production of Lactobacillus casei var. rhamnosus in milk whey
}

\author{
M. M. Alvarez, ${ }^{\star 1}$ E. J. Aguirre-Ezkauriatza, ${ }^{\star}$ A. Ramírez-Medrano, ${ }^{\star}$ and Á. Rodríguez-Sánchez† \\ *Centro de Biotecnologia-FEMSA, Tecnológico de Monterrey, Ave. Eugenio Garza Sada, 2501 sur. Monterrey, N.L., México \\ †Desarrolladora de Soluciones Sustentables (DSS), Ave. Eugenio Garza Sada, 2501 sur. Monterrey, N.L., México
}

\begin{abstract}
Lactobacillus casei is a lactic acid bacterium (LAB) that colonizes diverse ecological niches and that has found broad commercial application. The aim of this study was to characterize the kinetics of biomass production, lactic acid production, and substrate consumption of Lactobacillus casei var. rhamnosus cultured in deproteinized milk whey. Batch culture experiments were performed in an instrumented, 2-L, stirred tank bioreactor using different inoculum concentrations $(0.5$ to $1.0 \mathrm{~g} / \mathrm{L}$ ) and lactose levels (35 to $70 \mathrm{~g} / \mathrm{L}$ ). The time series of experimental data corresponding to biomass growth, lactose consumption, and lactic acid formation were differentiated to calculate the corresponding kinetic rates. Strong exponentially dependent product inhibition effects were evident at low lactic acid concentrations, and lactic acid production rate was partially associated with biomass growth. A mathematical model is presented that reproduces the experimental lactose, biomass, and lactic acid concentration profiles.
\end{abstract}

Key words: Lactobacillus casei var. rhamnosus, whey, kinetic model, bioreactor

\section{INTRODUCTION}

Lactobacillus casei is a lactic acid bacterium (LAB) that colonizes diverse ecological niches, including the human gastrointestinal tract (i.e., mouth and intestine). This organism is also highly diverse from the genetic standpoint (Cai et al., 2007) and many strains have found broad commercial applications. Because of the high capacity of $L$. casei to produce lactic acid, processoriented research has mainly focused on optimization and maximization of lactic acid production (see, for example, Kwon et al., 2001; Boudrant et al., 2005; Ding and Tan, 2006; John et al., 2007; Panesar et al., 2007; $\mathrm{Xu}$ et al., 2007). Currently only a few reports relate

Received January 27, 2010.

Accepted May 5, 2010.

${ }^{1}$ Corresponding author: Mario.alvarez@itesm.mx to the culture of probiotic biomass from L. casei in bioreactors (Mondragón-Parada and Nájera-Martinez, 2006; Aguirre-Ezkauriatza et al., 2010). For example, Mondragón-Parada and Nájera-Martinez (2006) suggested the possibility of producing $L$. casei biomass from whey-based medium supplemented with minimal amounts of yeast extract. However, these authors provided only limited kinetic information, derived exclusively from batch experiments at low lactose concentration. Recently, Aguirre-Ezkauriatza et al. (2010) have published data concerning the culture of a commercial L. casei strain on nonsupplemented goat milk whey, using batch and fed-batch bioreactors. Again, limited kinetic data were provided. In many countries, milk whey, derived largely from cheese processing, continues to be underutilized. In some instances, it is actually considered a serious ecological problem because of its high pollutant potential when improper disposal occurs. For example, in México, only $10 \%$ of generated whey is recovered and used, mainly for low-value applications (e.g., animal feeding). Whey has been used to grow LAB, again mainly for purposes of lactic acid production more than for biomass production (Lund et al., 1992; Shahbazi et al., 2005; Youssef and Goma, 2005; Altiok et al., 2006; Panesar et al., 2007; Agarwal et al., 2008). However, the current use of LAB as probiotic food supplements suggests that biomass production from whey wastes could be equally important.

In this context, the development of a simple, robust, scalable, and low-cost process capable of converting nonsupplemented whey into an added-value product is a highly attractive commercial proposition. However, there is limited kinetic knowledge that could help the engineer design, optimize, or scale up a lactic fermentation process. Several papers have been published relating to mathematical modeling or kinetic description of L. casei cultures (e.g., Youssef and Goma, 2005; Altiok et al., 2006; Aguirre-Ezkauriatza et al., 2010). Nevertheless, available models have not been derived from kinetic analysis of growth curves and experimental behaviors, but rather on an empirical fitting of parameters to experimental data. Indeed, the main objective of our contribution was to provide a simple model, based on 
experimental evidence, for lactic acid fermentations in whey, such that it could be used for process design or to assist in the establishment of operational process decisions. Here, we analyze the kinetics of biomass production, substrate consumption, and lactic acid production of Lactobacillus casei var. rhamnosus in deproteinized and unsupplemented milk whey. In particular, we characterize both the strong biomass growth inhibition that occurs due to product accumulation, as well as the lactic acid production rate with respect to the biomass growth rate. We propose a model that is consistent with relevant biological behaviors that we observed in our culture experiments of $L$. casei in nonsupplemented whey (e.g., a strong inhibitory effect of lactic acid on growth rate, and partial coupling of lactic acid production with respect to growth rate). The model adequately reproduces experimental growth and lactic acid production curves within a wide range of substrate conditions.

\section{MATERIALS AND METHODS}

\section{Whey Pretreatment}

Spray-dried milk whey, provided by Chilchota, Coahuila, México, was reconstituted by water addition to a final lactose concentration in the range of 35 to $70 \mathrm{~g} / \mathrm{L}$. Reconstituted whey was ultrafiltered using a Flexstand unit coupled to a Watson-Marlow 313S peristaltic pump, through an UFP-10-T-6A 10000 NMWC ultrafiltration column (surface area of $2800 \mathrm{~cm}^{2}$ ) from GE Healthcare Corp. (Westborough, MA). The filtrate was pasteurized at $80^{\circ} \mathrm{C}$ for $20 \mathrm{~min}$ in a Yamato sterilizer (model SE510, Yamato Scientific America, San Francisco, CA). No additional carbon or nitrogen source was added.

\section{Fermentation Experiments}

For all of the fermentation experiments reported here, a 3-L fully instrumented Applikon model EZ-Control bioreactor (Applikon Biotechnology, Schiedam, the Netherlands) was used. Experiments were run at $37^{\circ} \mathrm{C}$, $\mathrm{pH} 5.5$, and an agitation rate of $300 \mathrm{rpm}$. At selected time intervals, $25-\mathrm{mL}$ samples were removed from the bioreactor liquid to quantify biomass, lactic acid, and lactose concentrations. Results from 4 batch experiments are reported here. Experiments were run at different initial substrate concentrations, ranging from 35 to $70 \mathrm{~g} / \mathrm{L}$ of lactose $(35,42,50,55,57$, and $70 \mathrm{~g} / \mathrm{L})$. A food-grade commercial strain of freeze-dried Lactobacillus casei var. rhamnosus, from Sacco-Clerici (Cadorago, Italy) was used as inoculum. Experiments were run at inoculum concentrations of 0.5 and $1.0 \mathrm{~g} / \mathrm{L}$.

\section{Analytical Techniques}

Biomass content at different fermentation times was estimated by absorbance measurements at $560 \mathrm{~nm}$. Absorbance values were correlated to dry weight biomass values estimated from $15-\mathrm{mL}$ freeze-dried samples. Lactose, lactic acid, glucose, and galactose concentrations were measured by HPLC using a Waters chromatography system (model W1515) coupled to a refractive index detector (model W2414, Waters, Milford, MA). An Aminex HPX 87H (Bio-Rad, Hercules, CA) column and a $5 \mathrm{mM}$ sulfuric acid mobile phase were used at a flow rate of $0.6 \mathrm{~mL} / \mathrm{min}$ and temperatures of $50^{\circ} \mathrm{C}$ and $60^{\circ} \mathrm{C}$ in the detector and oven, respectively.

\section{Kinetics Analysis}

The biomass, lactose, and lactic acid concentration profiles observed in batch experiments were fitted to fourth-order polynomial equations using Kaleidagraph software (Synergy, Reading, PA). Polynomial equations were derived to calculate the experimental biomass production rate $\left(\mathbf{r}_{\mathrm{x}}\right.$, where $\mathrm{r}_{\mathrm{x}}=\mathrm{d}[\mathrm{X}] / \mathrm{dt}$, and $[\mathrm{X}]$ is biomass concentration), lactic acid production rate $\left(\mathbf{r}_{\mathrm{p}}\right.$, where $\mathrm{r}_{\mathrm{p}}=\mathrm{d}[\mathrm{P}] / \mathrm{dt}$, and $[\mathrm{P}]$ is lactic acid concentration), and lactose consumption rate $\left(\mathbf{r}_{\mathrm{s}}\right.$, where $\mathrm{r}_{\mathrm{s}}=\mathrm{d}[\mathrm{S}] / \mathrm{dt}$, and $[\mathrm{S}]$ is lactose concentration). Rates were analyzed to infer relationships between substrate, product, and biomass concentrations. A mathematical model consistent with these relationships was proposed. The kinetic parameters of the model were calculated from analysis of the experimental biomass, lactose and lactic acid profiles, and their production or consumption rates. The adequacy of the model was validated against 3 sets of experimental observations including biomass, lactose, and lactic acid data.

\section{Mathematical Model Formulation}

Here, we postulated that the material balances for $L$. casei var. rhamnosus biomass, substrate, and product (lactic acid) were, respectively,

$$
\begin{gathered}
\mathrm{r}_{\mathrm{x}}=\mathrm{d}[\mathrm{X}] / \mathrm{dt}=\mu[\mathrm{X}] \\
\mathrm{r}_{\mathrm{p}}=\mathrm{d}[\mathrm{P}] / \mathrm{dt}=\alpha \mathrm{r}_{\mathrm{x}}+\beta[\mathrm{X}] \\
\mathrm{r}_{\mathrm{s}}=-\mathrm{d}[\mathrm{S}] / \mathrm{dt}=\left(1 / \mathrm{Y}_{\mathrm{X} / \mathrm{S}}\right) \mathrm{r}_{\mathrm{x}}+\left(1 / \mathrm{Y}_{\mathrm{P} / \mathrm{S}}\right) \mathrm{r}_{\mathrm{p}} .
\end{gathered}
$$

Equation 1 states that the biomass production rate of L. casei in whey can be described by a general kinetic model of first order with respect to biomass, where $r_{x}$ is the rate of biomass production, $[\mathrm{X}]$ is the biomass 
concentration, $\mathrm{t}$ is the time, and $\mu$ is the specific growth rate. Equation 2 postulates that the rate of lactic acid production is affected by 2 contributions. The first reflects the association with the biomass growth rate through the proportionality constant $\alpha$. The second is proportional to biomass concentration and is mediated by the constant $\beta$. Equation 3 establishes that the negative accumulation at limiting substrate concentration (lactose) is only due to 2 processes: biomass growth $\left(\mathrm{r}_{\mathrm{x}}\right)$ and lactic acid production $\left(\mathrm{r}_{\mathrm{p}}\right)$. The biomass/substrate yield coefficient $\left(\mathrm{Y}_{\mathrm{X} / \mathrm{S}}\right)$ and the product/substrate yield coefficient $\left(\mathrm{Y}_{\mathrm{P} / \mathrm{S}}\right)$ define the relative weight of biomass growth and the lactic acid production on substrate consumption, respectively.

\section{Model Fitting to Experimental Data}

The proposed kinetic model was programmed in an Excel (Microsoft Corp., Redmond, WA) spreadsheet. Four experimental data sets were used to fit the mathematical model and to calculate the best set of kinetic parameters to properly reproduce the profiles of experimental biomass and lactic acid and lactose concentrations. These experimental data sets corresponded to 2 experiments of culture of $L$. casei var. rhamnosus growing in nonsupplemented whey, a previously reported data set of $L$. casei growing in unsupplemented goat whey (Aguirre-Ezkauriatza et al., 2010), and a set of experimental data of $L$. case $i$ growing in whey supplemented with $10 \mathrm{~g} / \mathrm{L}$ of yeast extract (data from Altiok et al., 2006).

\section{RESULTS AND DISCUSSION}

\section{Analysis of Biomass, Substrate, and Product Time Profiles}

Lactobacillus casei is a heterofermentative bacterium that mainly produces lactic acid. Other products include $\mathrm{CO}_{2}$, ethanol, and acetate. Figure 1 presents typical profiles of biomass production, lactose consumption, galactose accumulation, and lactic acid production of $L$. casei var. rhamnosus growing batchwise in deproteinized and unsupplemented milk whey. Three phases that correspond to different fermentation stages can be identified: an extended lag phase (Figure 1a), a biomass growth and lactic acid production phase (Figure 1b); and a lactic acid production stage with no further biomass accumulation (Figure 1c). Lag phases in the range of 5 to $10 \mathrm{~h}$ were typically observed in our experiments using freeze-dried inoculum. The extent of the lag phase inversely correlated with inoculum concentration. Shorter lag phases occurred (data not shown) in experiments where lactase was added at the

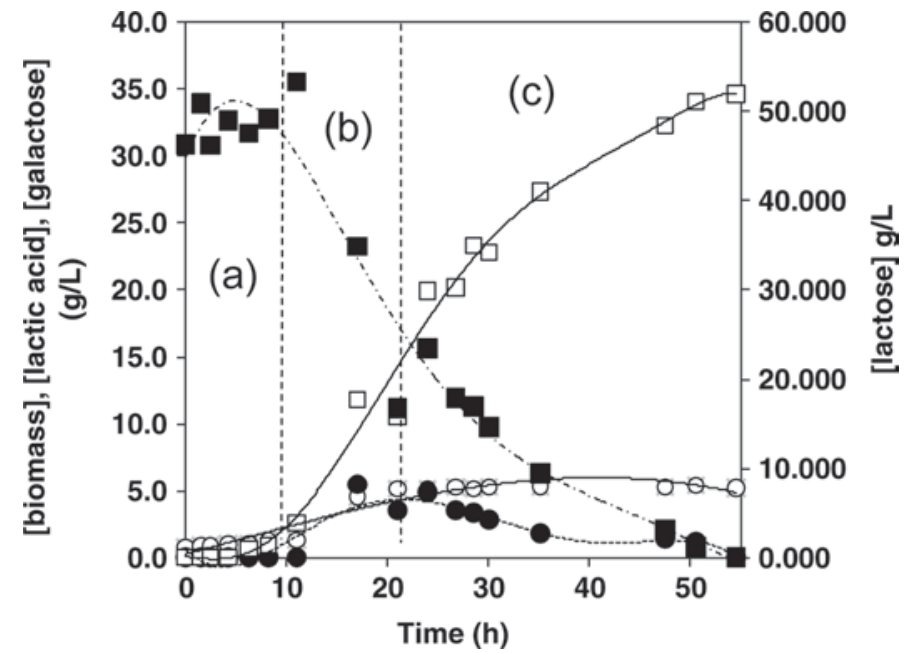

Figure 1. Biomass $(\bigcirc)$, lactose $(\boldsymbol{\square})$, lactic acid $(\square)$, and galactose $(\bullet)$ concentration profiles for a typical batch culture experiment of Lactobacillus casei var. rhamnosus in deproteinized and unsupplemented whey. Three culture stages can be differentiated: (a) lag phase period; (b) a phase of exponential biomass growth and lactic acid production; and (c) a stage where lactic acid continues to be produced but no further biomass growth is observed.

beginning of the fermentation. This result suggests that the extended lag phases observed are due to the time required for $L$. casei inoculum to adequately adapt its enzymatic machinery for lactose hydrolysis.

In a second fermentation stage, exponential biomass growth, lactic acid production, and galactose accumulation start simultaneously. Essentially no glucose accumulation was observed in our experiments, suggesting that glucose derived from lactose hydrolysis is immediately used. The galactose moiety, in contrast, did accumulate in the culture media (see Figure 1) during the early stages of the fermentation. This result suggests a relative preference of glucose over galactose during the period of rapid biomass production. The material balances on lactose, galactose, and glucose revealed that approximately $60 \%$ of galactose was used by $L$. casei during the first $15 \mathrm{~h}$ after the onset of exponential growth. By the end of the second fermentation stage (Figure 1b), biomass growth ceased. However, lactic acid production continued with a small but significant rate deflection, as evidenced by a change in slope observed in the lactic acid and lactose profiles at the beginning of the third fermentation stage (Figure 1c). Also, galactose consumption occurred at a faster rate, as evidenced by its concentration decrease in the culture media.

Later in this discussion, we will analyze in detail the relationships between biomass and lactose and lactic acid concentrations (and their respective rates of production or consumption) to infer the values of the ki- 


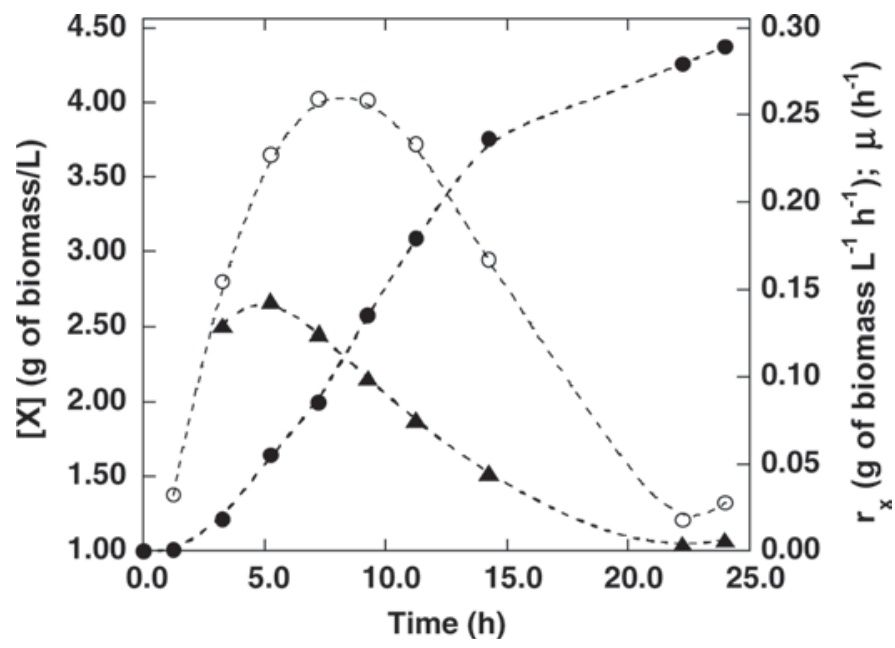

Figure 2. Time evolution of the biomass concentration ([X], •) the overall rate of biomass production $\left(\mathrm{r}_{\mathrm{x}}, \mathrm{O}\right)$, and the specific growth rate $(\mu, \boldsymbol{\Lambda})$, during the first $25 \mathrm{~h}$ of a batch culture experiment.

netic constants of the proposed model. The distinction between stages 2 and 3 of our fermentation experiments (Figure 1b and 1c) proved to be useful in conducting our kinetic analysis.

\section{Growth Rates Are a Strong Function of Lactic Acid Concentration}

To kinetically analyze the processes of $L$. casei var. rhamnosus growth, we fitted the experimental biomass concentration profile to a fourth-order polynomial trend, where a, b, c, and d are polynomial coefficients:

$$
[\mathrm{X}]=\mathrm{a}+\mathrm{bt}+\mathrm{ct} \mathrm{t}^{2}+\mathrm{d} \mathrm{t}^{3} .
$$

The analytical derivation of this polynomial function with respect to time allows for the calculation of the rate of biomass growth $\left(\mathrm{r}_{\mathrm{x}}\right)$ :

$$
\begin{gathered}
r_{x}=d[X] / d t=d\left[a+b t+c t^{2}+d t^{3}\right] / d t= \\
b+2 c t+3 d^{2} .
\end{gathered}
$$

Combining (Eq. [1]) and (Eq. [5]), the specific growth rate $(\mu)$ was calculated at each experimental point from

$$
\mathrm{r}_{\mathrm{x}}=\mu[\mathrm{X}] \text {; then } \mu=\left(\mathrm{b}+2 \mathrm{ct}+3 \mathrm{~d} \mathrm{t}^{2}\right) /[\mathrm{X}]
$$

Figure 2 presents the evolution of the biomass concentration $[\mathrm{X}]$ over time, the biomass growth rate $\left(\mathrm{r}_{\mathrm{x}}\right)$, and the specific growth rate $(\mu)$ for a batch experiment performed at $1 \mathrm{~g} / \mathrm{L}$ of inoculum. During the early stage of exponential growth (first $5 \mathrm{~h}$ in Figure 2), $\mu$ reached its highest values. However, because viable biomass concentration was at its lowest, the resulting biomass growth rate values were low. After $5 \mathrm{~h}$ of the experiment, the specific growth rate decreased substantially. Because the biomass concentration increased with time, $\mathrm{r}_{\mathrm{x}}$ peaked approximately half-way into the exponential growth phase. As the value of $\mu$ decreased further, the product $\mu[\mathrm{X}]$ also decreased, and $\mathrm{r}_{\mathrm{x}}$ eventually reached a zero value. After $15 \mathrm{~h}, \mu$ reached a value of $0.025 \mathrm{~h}^{-1}$ and continued to decrease to practically zero by the end of the exponential growth phase (after $20 \mathrm{~h}$ ). The biomass concentration, at its maximum, was unable to compensate for the effect of the steeper decrease in $\mu$. Consequently, biomass production also decreased and eventually ceased. The drastic decrease in the specific growth rate value is a signature feature of the growth behavior of lactic acid bacteria (Aguirre-Ezkauriatza et al., 2010) and can be attributed to the well-documented inhibitory effect of lactic acid accumulation.

Figure 3 shows the observed values of $\mu$ as a function of lactic acid concentration for 2 independent experiments run at different inoculum concentrations. In both cases, an exponential decay of the specific growth rate was observed with respect to lactic acid concentration. This behavior is consistent with a kinetic model of the type

$$
\mu=\mu_{0} \exp ^{(-\lambda[\mathrm{P}])}
$$

where $\lambda$ is defined as a product inhibition constant. With different degrees of severity, this behavior can be observed in other L. casei strains, in Lactobacillus helveticus, and in Lactococcus lactis (our unpublished data of work in progress). For the different inoculum and substrate concentrations tested for $L$. casei var. rhamnosus, the value of $\lambda$ is essentially the same, at $\lambda$ $=0.335 \pm 0.005 \mathrm{~L} / \mathrm{g}$. A consistent and robust direct estimation of the $\mu_{0}$ value is not as straightforward. Fitting of experimental data to equation [7] yielded values in the range of 0.20 to $2.4 \mathrm{~h}^{-1}$, depending on the substrate and initial inoculum concentration. Biologically, $\mu_{0}$ represents the value of the specific growth rate at the beginning of the exponential phase. As explained before, this occurs as a practically instantaneous value that changes exponentially as lactic acid production begins. A calculation based on fitting fourth-order polynomial equations for $[\mathrm{X}],[\mathrm{P}], \mathrm{r}_{\mathrm{x}}$, and $\mathrm{r}_{\mathrm{p}}$ results in a point-by-point estimation of $\mu$ versus $[\mathrm{P}]$ (Figure 3b). Fitting of these data to equation [7] results in estimates of $\lambda=0.340 \mathrm{~L} / \mathrm{g}$ and $\mu_{0}=2.38 \mathrm{~h}^{-1}$. As shown later, values of $\mu_{0}=2.0 \pm 0.2$ provided the best fit to 3 independent sets of experimental biomass and lactic acid 

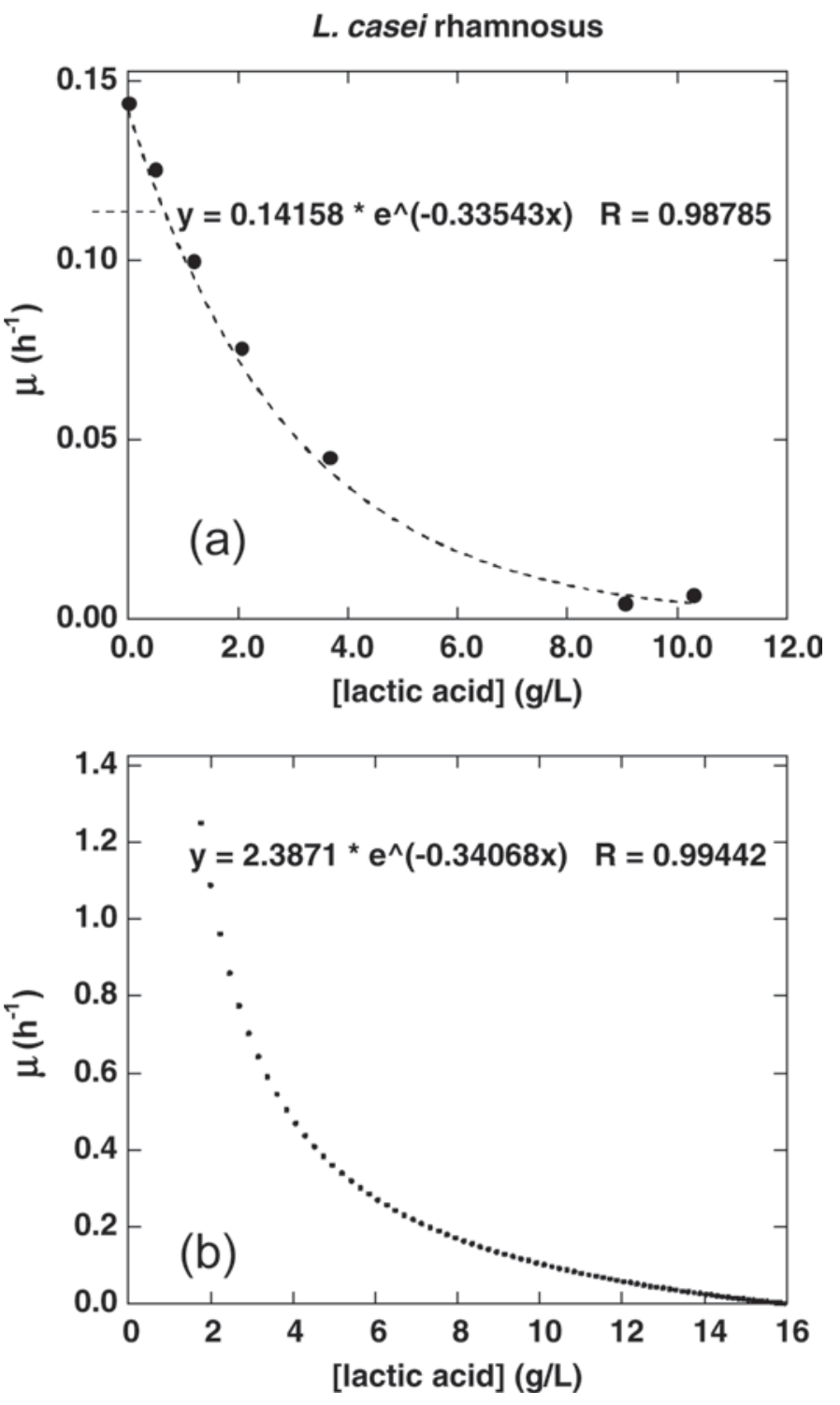

Figure 3. Dependence of the specific growth rate, $\mu(\bullet)$, with respect to lactic acid concentration in: (a) a batch fermentation experiment with an inoculum concentration of $[\mathrm{X}]_{0}=1.0 \mathrm{~g} / \mathrm{L}$; and $(\mathrm{b})$ a batch experiment at an inoculum concentration of $[\mathrm{X}]_{0}=0.5 \mathrm{~g} / \mathrm{L}$. Specific growth rate exhibits a strong dependence with respect to lactic acid concentration, which can be described by an exponential decay function. $[\mathrm{X}]_{0}=$ biomass concentration at time 0 (inoculation).

profiles. Using these parameter values in the simple kinetic model in equation [7], it was possible to accurately reproduce experimental biomass and lactic acid profiles without further need of introducing substrate dependence for the calculation of $\mu$. This exponential lactic acid inhibition effect has important practical process implications.

Figure 4 shows results from 5 batch fermentation experiments conducted at 2 different inoculum concentrations. When the net biomass production $\left([\mathrm{X}]-[\mathrm{X}]_{0}\right)$

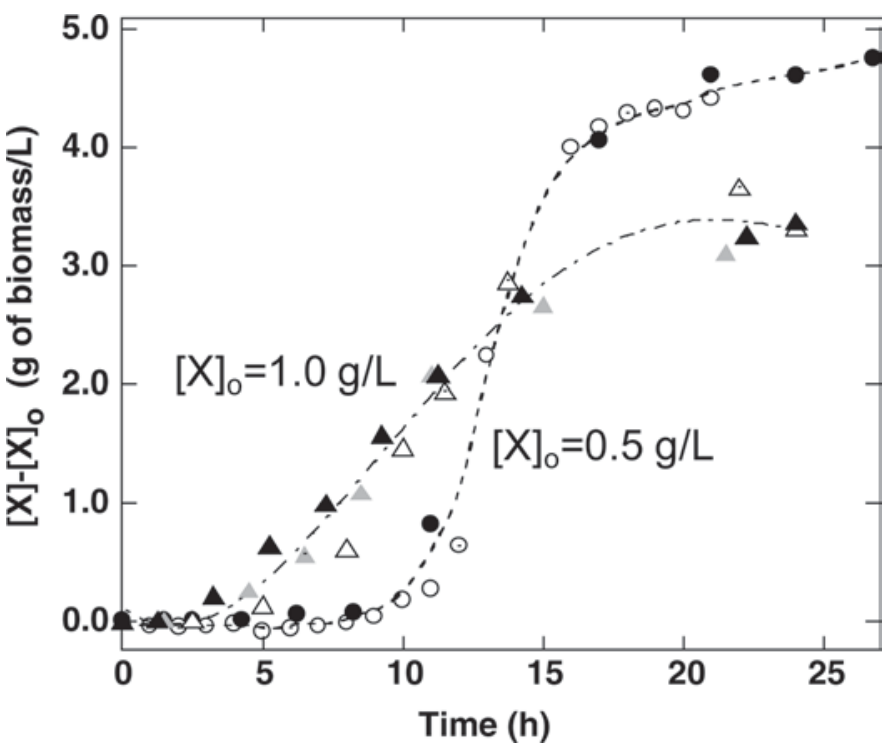

Figure 4. Biomass production $\left([\mathrm{X}]-[\mathrm{X}]_{0}\right)$ in time for the culture of Lactobacillus casei var. rhamnosus in nonsupplemented whey. Results are presented for batch experiments at 2 different inoculum concentrations: $[\mathrm{X}]_{0}=0.50 \mathrm{~g} / \mathrm{L}$ (circles) and $[\mathrm{X}]_{0}=1.0 \mathrm{~g} / \mathrm{L}$ (triangles) and different initial substrate concentrations (black, gray, and open symbols indicate experiments conducted at different initial lactose concentrations in the range from 35 to $70 \mathrm{~g} / \mathrm{L}$ ). Regardless of the initial substrate concentration, results from different experiments collapse into a master curve (---) depending on the inoculum concentration.

was plotted against time, all experimental curves collapsed into 2 master curves, depending on the initial inoculum concentration. For instance, the amount of inoculum used determined the final biomass concentration achieved. Counterintuitively, higher inoculum concentrations resulted in lower final biomass production. Higher initial biomass concentrations implied faster production and accumulation of lactic acid and the more severe inhibition effects resulted in a lower biomass net production.

\section{Lactic Acid Production Is Partially Associated with Growth}

Lactic acid production inhibits biomass growth. However, $r_{p}$ is also kinetically linked to biomass production rates and biomass concentrations. Figure 5 presents the time evolution of $r_{x}$ and $r_{p}$ for the first $12 \mathrm{~h}$ of a batch fermentation, corresponding to the stage of exponential growth (Figure 1b). The maxima for the curves appeared at different times. Even when $r_{x}$ had reached essentially a zero value, the lactic acid production rate still exhibited a positive value. Figure $5 \mathrm{~b}$ presents the evolution time frame for lactic acid concentration for the time period where the net biomass growth rate was basically zero $\left(\mathrm{r}_{\mathrm{x}} \sim 0\right.$; see also Figure $\left.1 \mathrm{c}\right)$. During this 

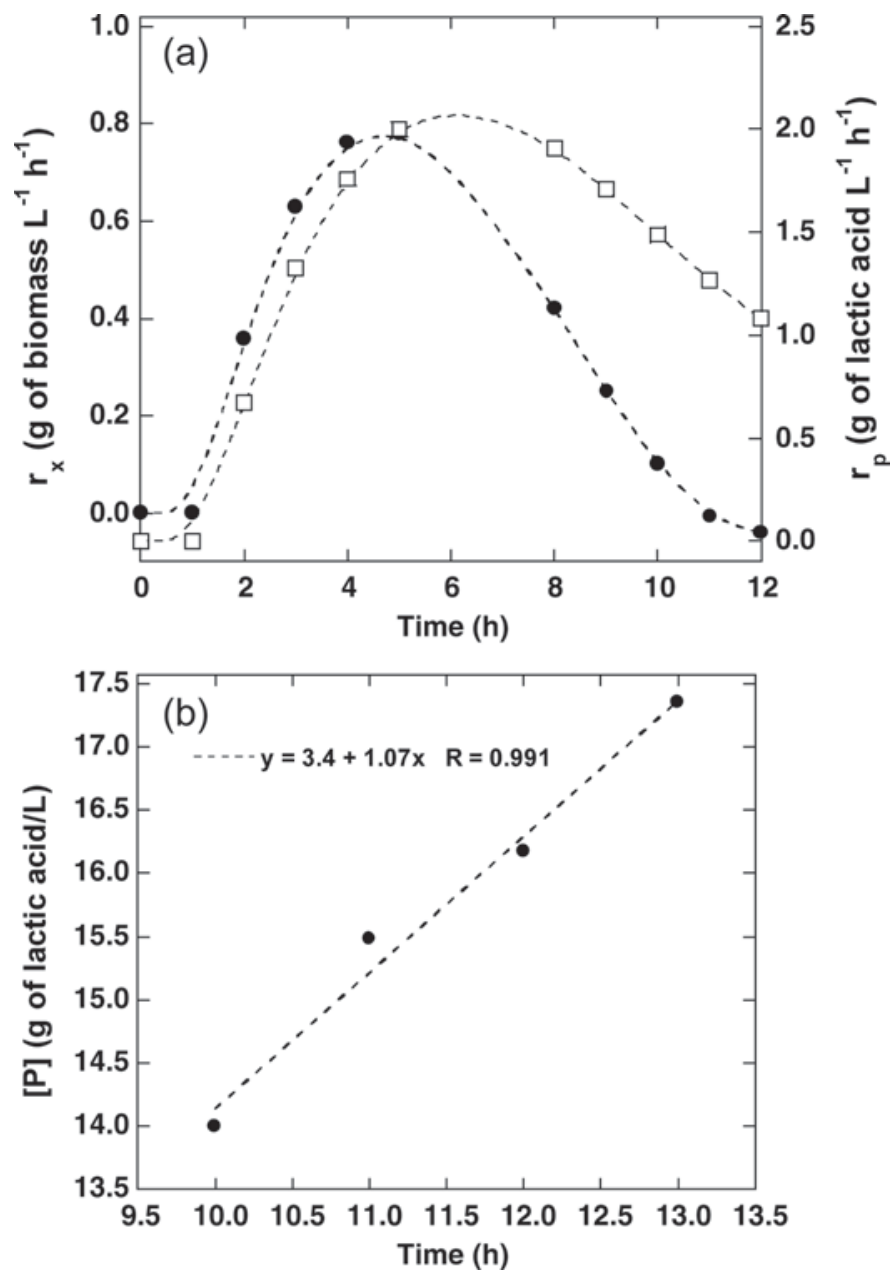

Figure 5. Lactic acid production is a function of both the biomass growth rate and the biomass concentration: (a) Time progression of the rate of lactic acid production $\left(\mathrm{r}_{\mathrm{p}}, \boldsymbol{\bullet}\right)$ and the rate of biomass growth $\left(\mathrm{r}_{\mathrm{x}}, \square\right)$ in a batch fermentation experiment, with an inoculum concentration of $[\mathrm{X}]_{0}=0.5 \mathrm{~g} / \mathrm{L}$. Both trends are similar in behavior, but not completely proportional. (b) During the stationary growth phase of the culture $\left(r_{x}=0\right)$, the increase in lactic acid concentration $([\mathrm{P}])$ is a linear function of time. The slope of $[\mathrm{P}]$ versus time curve is the rate of lactic acid production $\left(\mathrm{r}_{\mathrm{p}}\right)$ for this stage.

stage, the first term on the right side of equation [2] became negligible, therefore,

$$
\mathrm{r}_{\mathrm{p}}=\mathrm{d}[\mathrm{P}] / \mathrm{dt}=\beta[\mathrm{X}] .
$$

The slope of the straight line depicted in Figure 5b is given by $r_{p}=\beta[X]=1.07$. Because $[X]$ was constant at this fermentation stage, $\beta$ could be directly calculated as $\beta=0.19 \mathrm{~g}$ of lactic acid/g of biomass per hour. Knowing the $\beta$ value, equation [2] was used to estimate the value of $\alpha$ from the $r_{p}$ profiles calculated from polynomial fitting. From this analysis, an average value of $\alpha=1.8 \mathrm{~g}$ of lactic acid/g of biomass was
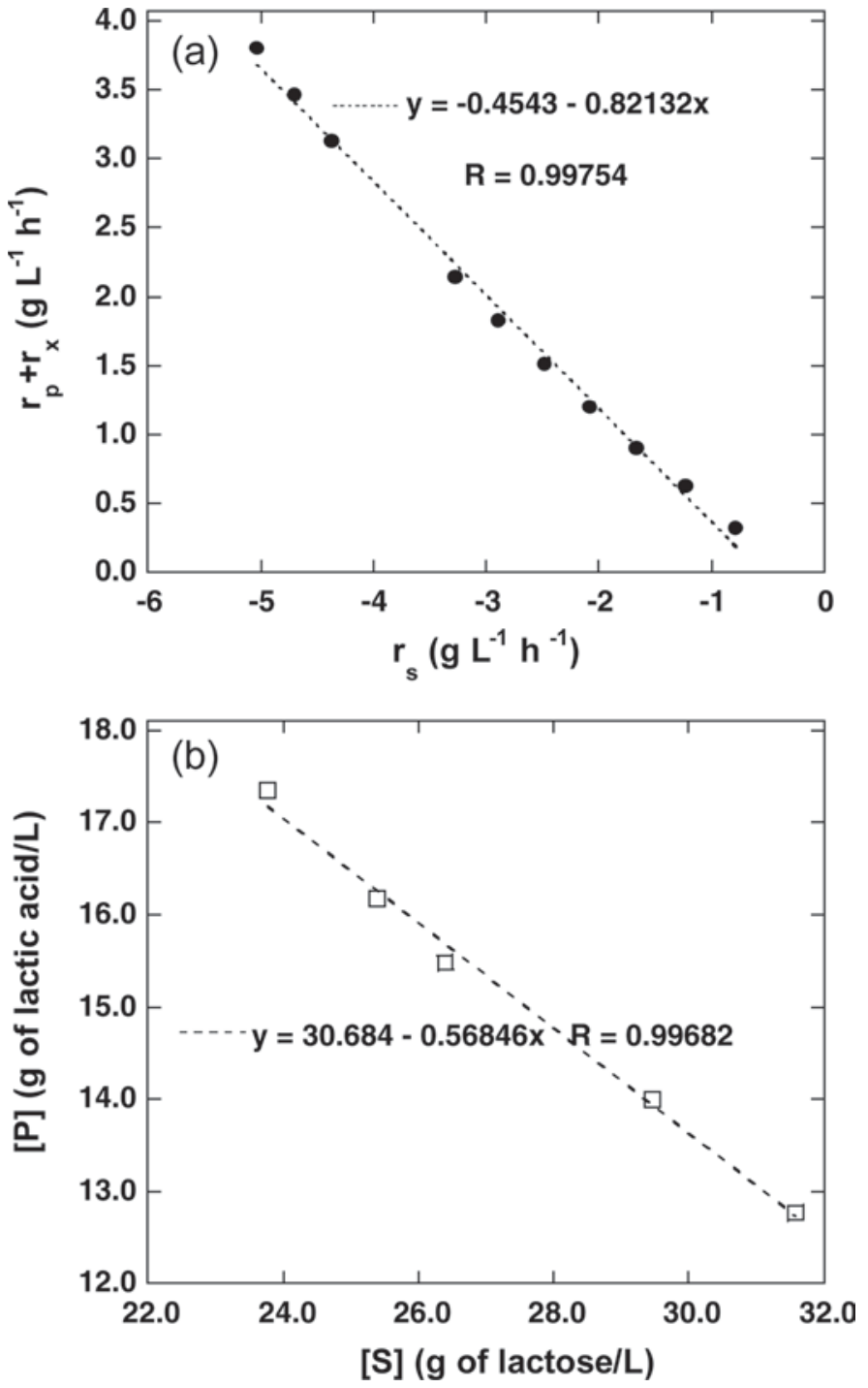

Figure 6. Lactose consumption is a function of 2 contributions: lactose consumed due to biomass growth and lactic acid production: (a) The sum of the rate of biomass production $\left(\mathrm{r}_{\mathrm{x}}\right)$ and lactic acid concentration $\left(\mathrm{r}_{\mathrm{p}}\right)$ is proportional to the rate of lactose consumption $\left(\mathrm{r}_{\mathrm{s}}\right)$. (b) The product/substrate yield coefficient $\left(\mathrm{Y}_{\mathrm{P} / \mathrm{S}}\right)$ can be evaluated from the slope of the straight line described when the lactic acid concentration $([\mathrm{P}])$ is plotted versus the lactose concentration ([S]) during the stationary growth phase of a batch fermentation experiment.

estimated. Therefore, lactic acid production in L. casei var. rhamnosus is partly growth associated.

The relative contribution of both terms in equation [2] is not equally significant during different stages of exponential growth. The $\alpha$ term dominates at the beginning of the exponential growth phase. Later, as the biomass concentration increases, the $\beta$ term becomes dominant. Once biomass growth stops, the lactic acid production rate becomes proportional to the active biomass concentration in the reactor. 
(a)

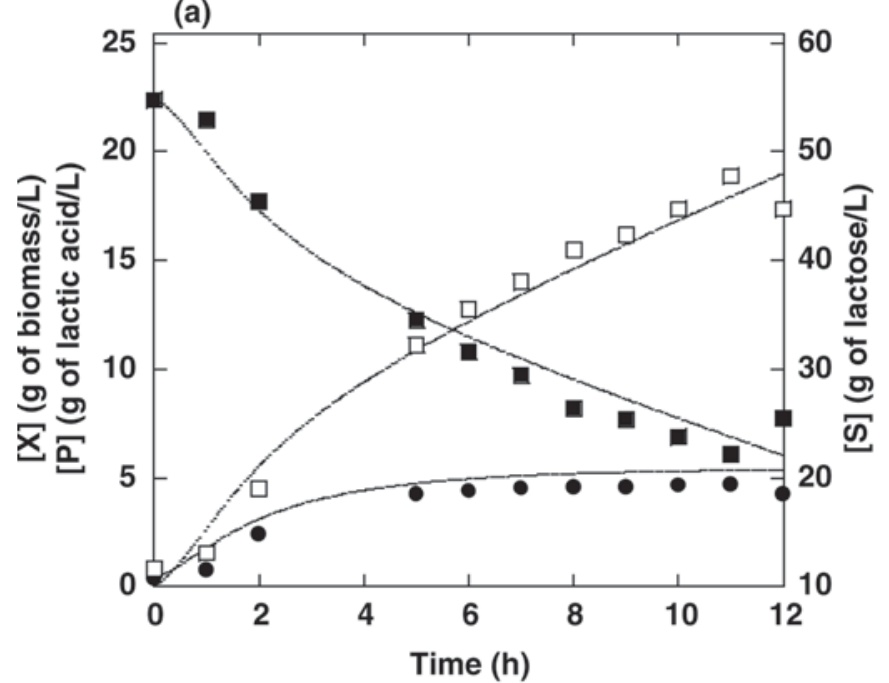

(b)

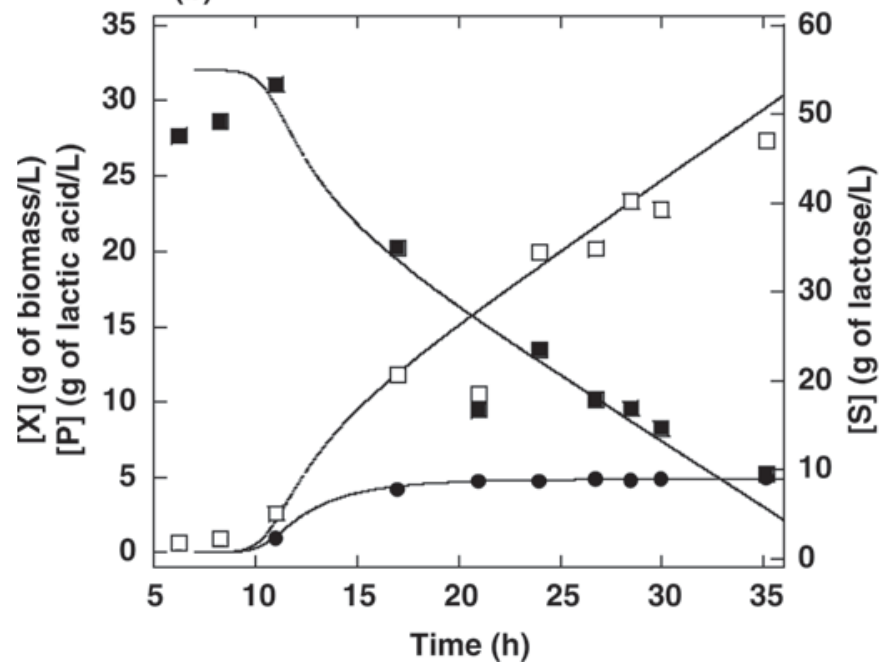

(c)

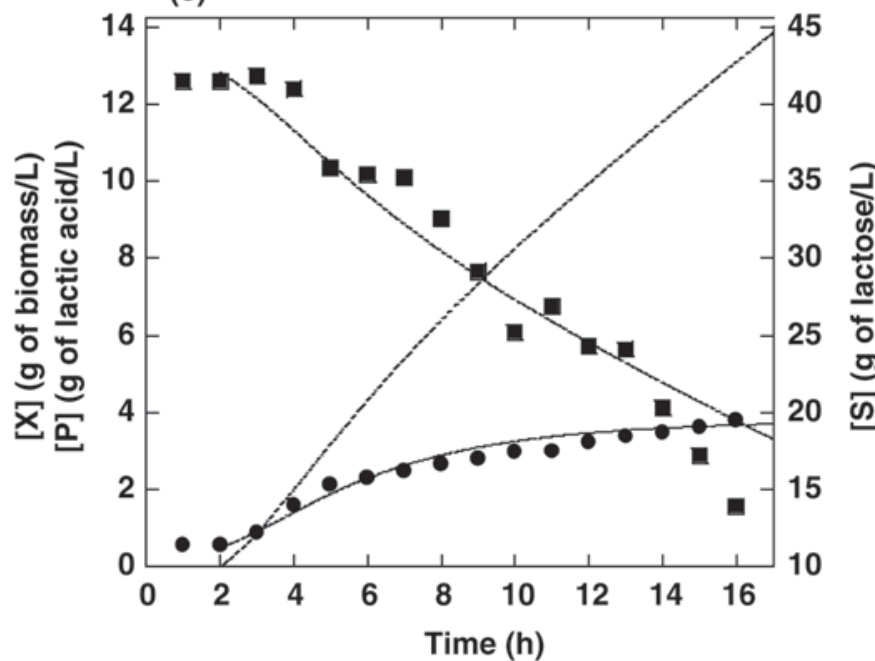

(d)

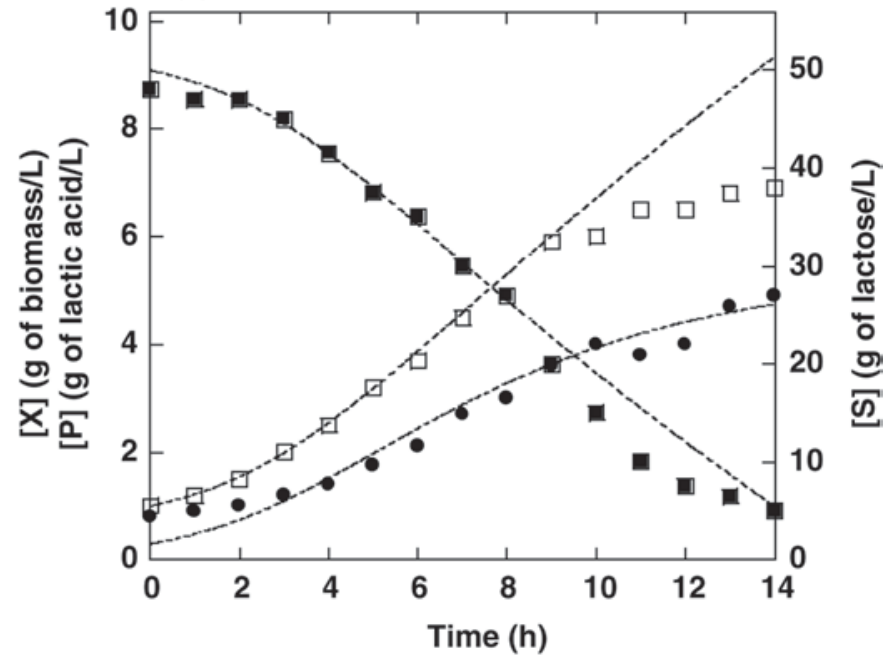

Figure 7. Simulation results $(-)$ from the proposed mathematical model and the kinetic parameters recommended in Table 1 adequately predict experimental biomass $([\mathrm{X}], \bullet)$, lactic acid $([\mathrm{P}], \square)$, and lactose $([\mathrm{S}], \mathbf{\square})$ concentration profiles with predictions in different batch culture experimental scenarios: (a) and (b) Lactobacillus casei var. rhamnosus growing in nonsupplemented whey (the current study); (c) L. casei growing in unsupplemented goat whey (data from Aguirre-Ezkauriatza et al., 2010); and (d) L. casei growing in whey supplemented with 10 g/L of yeast extract (data from Altiok et al., 2006).

\section{Estimation of Yield Coefficients $Y_{X / S}$ and $Y_{P / S}$}

At this point, the relationship between lactic acid production and biomass growth has been fully established. The relative contribution of these 2 processes on $r_{s}$ will be studied next. Equation [3] postulates that lactose consumption is only due to lactic acid production and biomass growth. Figure 6a demonstrates that, during the exponential growth phase, the sum of the rate of lactic acid production and the rate of biomass growth was proportional to the global rate of lactose consumption. The partial contribution of $r_{p}$ to $\mathrm{r}_{\mathrm{s}}$ was established by analysis of the nongrowth stage (see Figure 1c). Once biomass growth stops, $\mathrm{r}_{\mathrm{s}}$ become proportional to $\mathrm{r}_{\mathrm{p}}$ through the value of the product/ substrate yield coefficient $\left(\mathrm{Y}_{\mathrm{P} / \mathrm{S}}\right)$. Figure $6 \mathrm{~b}$ shows the relationship between lactic acid $([\mathrm{P}])$ and lactose $([\mathrm{S}])$ concentrations for the nongrowth fermentation stage. Indeed, in our batch experiments, this proportionality was observed, and $\mathrm{Y}_{\mathrm{P} / \mathrm{S}}$ was calculated from the slope of the straight line in Figure $6 \mathrm{~b}\left(\mathrm{Y}_{\mathrm{P} / \mathrm{S}}=0.5684\right)$. With this value, and using equation [3], the biomass/substrate yield coefficient $\left(\mathrm{Y}_{\mathrm{X} / \mathrm{S}}\right)$ can be estimated from the experimental data corresponding to the exponential growth stage. An average value for $\mathrm{Y}_{\mathrm{X} / \mathrm{S}}=1.5819 \pm$ 0.33 was observed. 
Table 1. Summary of kinetic parameters used to simulate biomass growth, lactic acid production, and lactose consumption of different Lactobacillus casei strains cultured in whey

\begin{tabular}{|c|c|c|c|c|}
\hline \multirow[b]{2}{*}{ Parameter $^{1}$} & \multirow[b]{2}{*}{ Unit } & \multicolumn{3}{|c|}{ Recommended parameter values } \\
\hline & & L. casei rhamnosus & L. casei $^{2}$ & L. casei $^{3}$ \\
\hline$\mu_{0}$ & $1 / \mathrm{h}$ & 2.2 & 0.7 & 0.7 \\
\hline$\lambda$ & $\mathrm{L} / \mathrm{g}$ of lactic acid & 0.34 & 0.34 & 0.34 \\
\hline$\alpha$ & $\mathrm{g}$ of lactic acid/g of biomass & 1.8 & 1.8 & 1.0 \\
\hline$\beta$ & $\mathrm{g}$ of lactic $\mathrm{acid} / \mathrm{h}$ & 0.193 & 0.193 & 0.100 \\
\hline $\mathrm{Y}_{\mathrm{X} / \mathrm{S}}$ & $\mathrm{g}$ of biomass / $\mathrm{g}$ of lactose & 1.582 & 1.582 & 1.582 \\
\hline $\mathrm{Y}_{\mathrm{P} / \mathrm{S}}$ & $\mathrm{g}$ of lactic acid/g of lactose & 0.638 & 0.638 & 0.200 \\
\hline
\end{tabular}

\section{Mathematical Modeling}

The kinetic parameters estimated in previous sections, together with the kinetic model presented in equations [1], [2], [3], and [7], were used to simulate batch culture experiments of $L$. casei var. rhamnosus in deproteinized and nonsupplemented whey. A comparison of simulation and experimental results is presented in Figures $7 \mathrm{a}$ and 7b. Table 1 presents the set of parameter values used in these simulations. In general, for all of the experimental sets analyzed, the model accurately reproduces the biomass, lactose, and lactic acid profiles in the range of inoculum concentrations covered experimentally $(0.5$ to $1.0 \mathrm{~g} / \mathrm{L}$ ) and over a wide range of lactose concentrations $(25$ to $70 \mathrm{~g} / \mathrm{L})$. However, the model overestimated lactic acid production and lactose consumption in regions where lactose concentration was $<20 \mathrm{~g} / \mathrm{L}$. This observation indicates that lactic acid production begins to be limited by substrate availability at low substrate concentrations and suggests that, in this concentration regimen, the lactic acid production rate becomes a function of lactose concentration; that is, $r_{p}=r_{p}[S]$.

In addition, the model was used to reproduce 2 sets of experimental biomass and lactose profiles that have been previously reported by our group (Aguirre-Ezkauriatza et al., 2010) for a commercial L. casei strain growing in goat milk whey. By varying $\mu_{0}$ and keeping the rest of the parameters constant, a good fit with the experimental data was obtained (Figure 7c). The model was also validated against experimental data reported by Altiok et al. (2006). In these experiments, $L$. case $i$ was used to ferment whey supplemented with yeast extract $(10 \mathrm{~g} / \mathrm{L})$ and with an initial nonzero lactic acid concentration. A comparison between the reported data and our simulations is shown in Figure 7d. By using the set of parameters specified in the last column in Table 1, biomass and lactic acid concentration profiles were closely followed during the first $10 \mathrm{~h}$ of fermentation. As before, for the last stage of the fermentation, when the lactose concentration was $<20 \mathrm{~g} / \mathrm{L}$, the model overestimated lactic acid production.

\section{CONCLUSIONS}

In summary, we experimentally studied the kinetics of lactic acid production, biomass growth, and lactose consumption during batch culture of $L$. casei var. rhamnosus in deproteinized and nonsupplemented milk whey. The strong inhibitory effect of lactic acid on the biomass growth rate was characterized and described by a simple kinetic model, where the specific growth rate exponentially decayed as lactic acid accumulated. Lactic acid production was partly associated with biomass growth. The overall rate of lactose consumption can be described by the simple sum of the contributions of substrate consumption needed for biomass growth and substrate consumption required for lactic acid production. Based on our analysis, a mathematical model was derived and its kinetic parameters were established. The model accurately describes lactose, lactic acid, and biomass concentration profiles in $L$. casei var. rhamnosus batch fermentation experiments in whey milk. By varying some of the kinetic parameter values recommended for L. casei var. rhamnosus, the model was capable of describing the corresponding profiles for other L. casei strains. This suggests that the proposed model may be useful for generic prediction of the performance of different $L$. casei strains in whey.

\section{ACKNOWLEDGMENTS}

This study was funded by DSS México and Tecnológico de Monterrey (through the research chair CAT122). 


\section{REFERENCES}

Agarwal, L., K. Dutt, G. K. Meghwanshi, and R. K. Saxena. 2008. Anaerobic fermentative production of lactic acid using cheese whey and corn steep liquor. Biotechnol. Lett. 30:631-635.

Aguirre-Ezkauriatza, E. J., J. M. Aguilar-Yáñez, A. Ramírez-Medrano, and M. M. Alvarez. 2010. Production of probiotic biomass (Lactobacillus casei) in goat milk whey: Comparison of batch, continuous and fed-batch cultures. Bioresour. Technol. 101:2837-2844.

Altiok, D., F. Tokatli, and S. Harsa. 2006. Kinetic modelling of lactic acid production from whey by Lactobacillus casei (NRRL B-441). J. Chem. Technol. Biotechnol. 81:1190-1197.

Boudrant, J., N. V. Menshutina, A. V. Skorohodov, E. V. Guseva, and M. Fick. 2005. Mathematical modelling of cell suspension in high cell density conditions - Application to L-lactic acid fermentation using Lactobacillus casei in membrane bioreactor. Process Biochem. 40:1641-1647.

Cai, H., B. T. Rodriguez, W. Zhang, J. F. Broadbent, and J. L. Steele. 2007. Genotypic and phenotypic characterization of Lactobacillus casei strains isolated from different ecological niches suggests frequent recombination and niche specificity. Microbiology 153:2655-2665.

Ding, S. F., and T. W. Tan. 2006. L-Lactic acid production by Lactobacillus casei fermentation using different fed-batch feeding strategies. Process Biochem. 41:1451-1454.

John, R. P., K. M. Nampoothiri, and A. Pandey. 2007. Polyurethane foam as an inert carrier for the production of $\mathrm{L}(+)$-lactic acid by Lactobacillus casei under solid-state fermentation. Lett. Appl. Microbiol. 44:582-587.

Kwon, S., I.-K. Yoo, W. G. Lee, H. N. Chang, and Y. K. Chang. 2001. High-rate continuous production of lactic acid by Lactobacillus rhamnosus in a two-stage membrane cell-recycle bioreactor. Biotechnol. Bioeng. 73:25-34.

Lund, B., B. Norddahl, and B. Ahring. 1992. Production of lactic acid from whey using hydrolysed whey protein as nitrogen source. Biotechnol. Lett. 14:851-856.

Mondragón-Parada, M. E., and M. Nájera-Martinez. 2006. Lactic acid bacteria production from whey. Appl. Biochem. Biotechnol. 134:223-232.

Panesar, P. S., J. F. Kennedy, C. J. Knill, and M. R. Kosseva. 2007. Applicability of pectate-entrapped Lactobacillus casei cells for $\mathrm{L}(+)$ lactic acid production from whey. Appl. Microbiol. Biotechnol. $74: 35-42$.

Shahbazi, A., M. R. Mims, Y. B. Li, V. Shirley, S. A. Ibrahim, and A. Morris. 2005. Lactic acid production from cheese whey by immobilized bacteria. Appl. Biochem. Biotechnol. 121-124:529-540.

Xu, Z., Q. H. Wang, P. Wang, G. Cheng, Y. Ji, and Z. Jiang. 2007. Production of lactic acid from soybean stalk hydrolysate with $L a c$ tobacillus sake and Lactobacillus casei. Process Biochem. 42:8992.

Youssef, C. B., and G. Goma. 2005. Kinetic modeling of Lactobacillus casei ssp. rhamnosus growth and lactic acid production in batch cultures under various medium conditions. Biotechnol. Lett. $27: 1785-1789$ 